\title{
Efficient embedding network for 3D brain tumor segmentation
}

\author{
Hicham Messaoudi ${ }^{1}$, Ahror Belaid ${ }^{1}$, Mohamed Lamine Allaoui ${ }^{1}$, Ahcene \\ Zetout $^{1}$, Mohand Said Allili ${ }^{2}$, Souhil Tliba ${ }^{1,3}$, Douraied Ben Salem ${ }^{4,5}$, and \\ Pierre-Henri Conze ${ }^{4,6}$ \\ 1 Medical Computing Laboratory (LIMED), University of Abderrahmane Mira, \\ 06000, Bejaia, Algeria, http://http://www.univ-bejaia.dz/limed/ \\ ahror.belaid@univ-bejaia.dz \\ 2 Université du Québec en Outaouais, Gatineau, Québec, J8X 3X7 \\ 3 Neurosurgery Department, University Hospital Center, \\ Biological Engineering of Cancers, 06000 Bejaia, Algeria \\ ${ }^{4}$ Laboratory of Medical Information Processing (LaTIM) \\ UMR 1101, Inserm, 22 avenue Camille Desmoulins, 29238 Brest, France \\ 5 Neuroradiology Department, CHRU la cavale blanche, \\ Boulevard Tanguy-Prigent, 29609 Brest, France \\ 6 IMT Atlantique, Technopôle Brest Iroise, 29238 Brest , France
}

\begin{abstract}
D medical image processing with deep learning greatly suffers from a lack of data. Thus, studies carried out in this field are limited compared to works related to $2 \mathrm{D}$ natural image analysis, where very large datasets exist. As a result, powerful and efficient 2D convolutional neural networks have been developed and trained. In this paper, we investigate a way to transfer the performance of a two-dimensional classification network for the purpose of three-dimensional semantic segmentation of brain tumors. We propose an asymmetric U-Net network by incorporating the EfficientNet model as part of the encoding branch. As the input data is in $3 \mathrm{D}$, the first layers of the encoder are devoted to the reduction of the third dimension in order to fit the input of the EfficientNet network. Experimental results on validation and test data from the BraTS 2020 challenge demonstrate that the proposed method achieve promising performance.
\end{abstract}

Keywords: Convolutional encoder-decoders · Embedding networks . Transfer learning $\cdot 3 \mathrm{D}$ image segmentation · EfficientNet

\section{Introduction}

Gliomas are the most common type of primary brain tumors of the central nervous system. They can be of low-grade or high-grade type. High-Grade Gliomas (HGG) are an aggressive type of malignant brain tumors that grow rapidly. Furthermore, Low-Grade Gliomas (LGG) are classified into grade I and grade II. These tumors represent less than $50 \%$ of glial tumors and are considered as isolated tumor cells within the nervous parenchyma, with slow initial growth. 
Gliomas are also characterized by their infiltrating character resulting in ambiguous and fuzzy boundaries.

However, the treatment of LGG remains difficult due to the variability of tumor size, location, histology and biological behavior. Furthermore, because of the pressure that the tumor exhibits, normal tissues get deformed, making it even harder to distinguish normal tissues from tumoral areas. The diagnosis of these tumors followed by early treatments are critical for patient survival. Indeed, in most cases, patients who are suffering from LGG die in the next ten years of the initial diagnosis. For all these reasons, accurate and reproducible segmentation of gliomas is a pre-requisite step for investigating brain MRI data.

Magnetic Resonance Imaging (MRI) has been quickly imposed as being an essential medical imaging modality for disease diagnosis. MRI is particularly useful for brain tumor diagnosis, patient follow-up, therapy evaluation and human brain mapping $[5,15,11]$. The main advantage related to the use of MRI is its ability of acquiring non-invasive and non-irradiant medical images. It is also very sensitive to the contrast and provides an excellent spatial resolution which is entirely appropriate for the exploration of the brain tissues nature. In addition, the imaging easily derives $3 \mathrm{D}$ volumes according to brain tissues.

The multimodal Brain Tumor Segmentation (BraTS) challenge $[8,3,1,2,4]$ aims at encouraging the development of state-of-the-art methods for the segmentation of brain tumors by providing a large 3D MRI dataset of annotated LGG and HGG. The BraTS 2020 training dataset include 369 cases (293 HGG and 76 LGG), each with 4 modalities describing: native (T1), post-contrast T1-weighted (T1Gd), T2-weighted (T2), and T2 Fluid Attenuated Inversion Recovery (T2FLAIR) volumes, which were acquired with different clinical protocols and various MRI scanners from multiple $(\mathrm{n}=19)$ institutions. Each tumor was segmented into edema, necrosis and non-enhancing tumor, and active/enhancing tumor. Annotations were combined into 3 nested sub-regions: Whole Tumor (WT), Tumor Core (TC) and Enhancing Tumor (ET).

In the last decade, Convolutional Neural Networks (CNNs) have outperformed all others traditional methodologies in biomedical image segmentation. Particularly, the U-Net architecture [10] is currently experiencing a huge success, as most winning contributions to recent medical image segmentation challenges were exclusively build around U-Net.

The aim of this work is to investigate how to re-use powerful deep convolutional networks that exist in the literature for $2 \mathrm{D}$ image analysis purposes. Indeed, a lot of powerful 2D classification networks are well trained on very large datasets. Through transfer learning, these pre-trained networks can be easily reused for other classification problems. However, transferring both learning and feature detection power of classification networks to another types of problem is not obvious. Especially, in problems using convolutional encoder-decoder architectures such as U-Net, this consists in integrating the pre-trained model as part of the encoder branch. When the dimensions of the classifier and the processed network are the same (i.e. when they processed data having the same dimensions), the integration of the classifier is almost immediate. Otherwise, an 
adaptation process is necessary to be able to adapt the classifier to deal with the dimensions of the images under study.

Related works can be found in the literature where the segmentation of 3D medical images are based on 2D networks whose encoder has been pre-trained on ImageNet $[6,7,13]$. Although taking advantage of a pre-trained encoder, these networks do not integrate the spatial coherence in $3 \mathrm{D}$, since they process data slice by slice. In our case, we aim at studying how to transfer the skills of a powerful and pre-trained network on 2D images to a convolutional encoderdecoder processing 3D images without loosing the consistency across the third dimension. It is known that the field of $2 \mathrm{D}$ image classification is well studied and well developed, generally because of the availability of large annotated 2D natural image datasets. The public availability of such large 3D databases is non-existent, and even less so in the medical field. Taking advantage of such powerful and well-studied networks by re-using them in a 3D problem is an idea worth investigating.

In summary, the main proposed contributions are :

- Proposal of an efficient way to transfer any 2D classification architecture to $3 \mathrm{D}$ segmentation purposes without losing the $3 \mathrm{D}$ consistency.

- The proposed idea is generalizable in order to integrate any low-dimensional classification architecture into another high-dimensional architecture without losing spatial coherence.

In particular, we are interested in the recently published EfficientNet network [12] which achieves state-of-the-art top-1 and top-5 accuracy on ImageNet, while being widely more smaller and faster on inference than the best existing deep architectures. This paper is organized as follow: the proposed architecture is detailed in the next section (2), the results are summarized in section 3 , followed by a conclusion in section 4 .

\section{Method}

The proposed segmentation approach follows a convolutional encoder-decoder architecture. It is built from an asymmetrically large encoder to extract image features and a smaller decoder to reconstruct segmentation masks. We embed as part of the encoder branch the recently proposed network called EfficientNet [12].

\subsection{Data pre-processing}

Because of the limitations in GPU memory and time-consuming computation, we were forced to take some precautions. We process each modality separately, and resize the images dimensions by reducing the background using the largest crop size of $192 \times 160 \times 108$, and compromise the batch size to be 1 . We do not use any additional training data and employ the provided training set only. We normalize all input images to have zero mean and unit variance. 


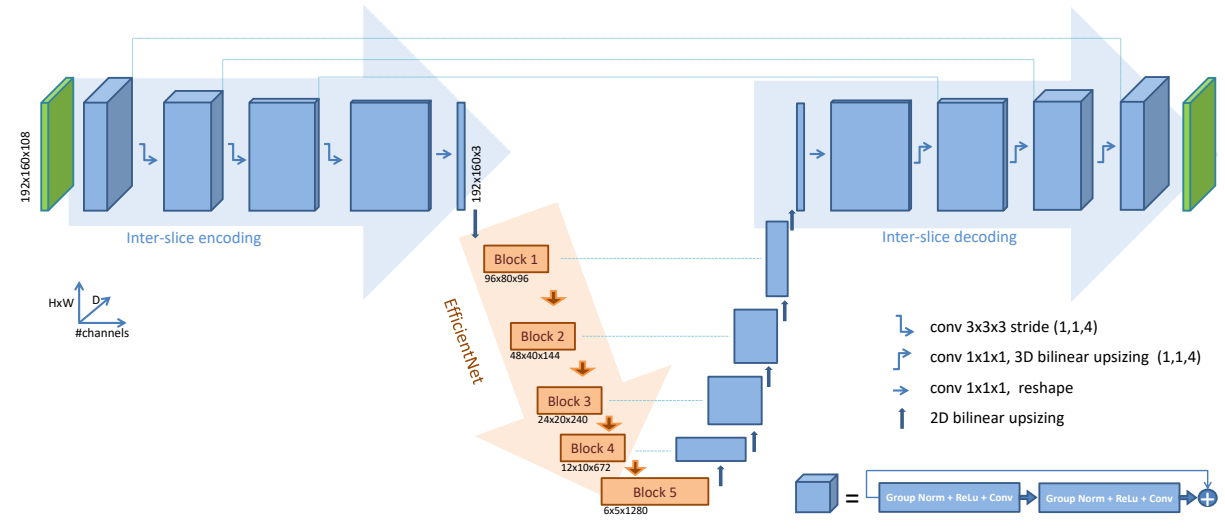

Fig. 1. Schematic illustration of the proposed network architecture. Input is a onechannel cropped 3D MRI. The inter-slice encoder as well as the decoder consist of a succession of residual blocks with GroupNorm normalization. The output of the decoder has three channels with the same spatial size as the input. Under each EfficientNet block are shown corresponding output feature dimensions.

Since the EfficientNet models range from 0 to 7 , we restricted our experimentations in this preliminary work only by testing the baseline EfficientNet-B0 [12]. Indeed, this model presents a compromise between performance and complexity. Using EfficientNet-B7 at this stage requires more resources, a choice which may strongly complexify our model. In any case, if the proposed architecture works well with this baseline model, its generalization towards EfficientNet-B7, will be straightforward and will definitely improve the performances.

\subsection{Encoder branch}

The encoding process goes through two steps. First, we encode three-dimensional data into two-dimensional data, while keeping the height and width at their original size and compressing only the depth to 3 channels. Second, the data is now ready to start the second encoding step, which is none other than the EfficientNet network without its fully connected layers.

As shown in Fig. 1, EfficientNet is represented as blocks as in its original version. However, only the blocks involved in skip connection layers are represented. The inter-slices encoding part uses convolutional blocks which consists of two convolutional layers with normalization and ReLU, followed by skip connection. Following the works of [9], we choose to use Group Normalization [14], which divides the channels into groups before normalizing them by using mean and variance of each group. It seems to perform better than traditional batch normalization, especially when the batch size is small.

Let us assume that the input volume is of width $\mathrm{W}$, height $\mathrm{H}$, depth $\mathrm{D}$, with $\mathrm{C}$ channels. The data passes through a 3D-2D shrinking step. Thus, the depth is reduced by factors 3,3 and 4 to reach a final depth size of 3 , which 
corresponds to the required number of channels of EfficientNet. In this shrinking procedure, the width and height of the single 3D batch are not modified. The different depth reduction factors can be changed and adapted according to the third dimension of the data. In our study, dimensions change as shown in Tab. 1. After this process, the reduced data reached through the $2 \mathrm{D}$ shrinking process are given as inputs of the EfficientNet model.

\begin{tabular}{ll}
\hline Layer & Dimension \\
\hline Input & $W \times H \times D \times C$ \\
block 1 & $W \times H \times \frac{D}{f_{1}} \times C_{1}$ \\
block 2 & $W \times H \times \frac{D}{f_{2}} \times C_{2}$ \\
$\vdots$ & $\vdots$ \\
block n & $W \times H \times 3 \times C_{n}$ \\
block n+1 & $W \times H \times 3 \times 1$ \\
output & $W \times H \times 3$ \\
\hline
\end{tabular}

Table 1. Dimension shrinking.

\subsection{Decoder branch}

Asymmetrically to the encoding part, the decoder is composed entirely of homogeneous blocks as shown in Fig. 1. Obviously, the decoding part linked to the EfficientNet is a 2D decoder whereas the inter-slice decoding is a 3D decoder. Each decoder level begins by upsampling the spatial dimension, doubling the number of features by a factor of 2 followed by skip connections. A sigmoid function is used as an activation for the output of the decoder which has three channels corresponding to the number of classes with the same spatial size as inputs.

\subsection{Loss}

Many networks are trained with a cross-entropy loss function, however the resulting delineations may not be ideal in terms of Dice score. As an alternative, one can employ a soft Dice loss function to train the proposed network. While several formulations of the dice loss exist in the literature, we prefer to use a soft Dice loss which has given good results in segmentation challenges in the past [9]. The soft Dice loss function is differentiable and is given by:

$$
\mathcal{L}_{\text {Dice }}=\frac{2 \sum P_{\text {true }} P_{\text {pred }}}{\sum P_{\text {true }}^{2}+\sum P_{\text {pred }}^{2}+\epsilon},
$$


were $P_{\text {true }}$ and $P_{\text {pred }}$ represent respectively the ground truth and the predicted labels. Brain MRI segmentation is a challenging task partly due to severe class imbalance. Tackling this issue by only using a fixed loss function, cross entropy or Dice, for the entire training process is not an optimal strategy. Therefore, a linear combination of the two loss functions is often considered as the best practice, and leads to more robust and optimal segmentation models. In practice, the final loss function is as follows:

$$
\mathcal{L}=\mathcal{L}_{\text {Cross }}-\mathcal{L}_{\text {Dice }}
$$

\subsection{Training}

The proposed network architecture is trained with centered cropped data of size $192 \times 160 \times 108$ voxels, ensuring that the useful content of each slice remains within the boundaries of the cropped area, training was made on BraTS2020 [8] dataset. Constrained by the poor performance of the material, we set the batch size to 1. Training has been done using the Adam optimizer known for little memory requirements, with an initial learning rate of $10^{-4}$ reduced by a factor of 10 whenever the loss has not improved for 50 epochs.

\section{$3 \quad$ Results}

We designed the proposed network on Tensorflow and run it on the 368 training cases of BraTS 2020. We process the four modalities separately and average the sigmoid outputs. The validation dataset was provided to test the performance of the models on unseen data. It consists of 125 cases with 4 modalities and without their corresponding segmentation. The results of the segmentation of the Whole Tumor (WT), Tumor Core (TC) and Enhancing Tumor (ET) are summarized in Tab. 2. All reported values were computed by the online evaluation platform (https://ipp.cbica.upenn.edu/). Fig. 2 shows a typical segmentation results extracted from the validation dataset.

With an average Dice score of 84.13 for the WT class, on the validation set, the proposed model seems efficient and accurate enough to handle the training and inference over a 3D dataset. On the other hand, results on enhanced and core tumors are less efficient. This may be due to the fact that we process each MRI modality separately.

After the compression on the depth, the network retains the shape of the brain and its structure, even if they appear slightly blurred and degraded. The network learns to extract three axial sections at more or less regular levels. We notice the presence of the tumor on the three learned sections, the axial sections generally involved in 3-channel compression are the sections that denote the presence of tumor information. The high intensity on the tumor parts indicates that the features of the network are focused on the detection of the tumor. These details are reported in Fig. 3 and Fig. 4.

The results on the test set are reported on Tab. 3. We can notice a large improvement on ET and TC scores and a slight decrease on WT compared 
to the validation set. From Fig. 3, we can see that the images resulting from the $3 \mathrm{D}$ compression of the axial slices are well oriented on the learning of the characteristics of the whole tumor, thus the visible region on FLAIR. In Fig. 4 we find that the compressed data are much more oriented in learning the structural characteristics of enhanced and necrotic tumor components, which are the visible regions in $\mathrm{T} 1 \mathrm{CE}$.

\begin{tabular}{|c|c|c|c|}
\hline & \multicolumn{3}{|c|}{ Dice } \\
\hline & $\mathrm{ET}$ & WT & $\mathrm{TC}$ \\
\hline Mean & 65.37 & 84.13 & 68.04 \\
\hline StdDev & 31.93 & 10.67 & 31.29 \\
\hline Median & 81.23 & 87.08 & 78.30 \\
\hline
\end{tabular}

Table 2. Results on BraTS 2020 validation data. Metrics were computed by the online evaluation platform.

\begin{tabular}{|c|c|c|}
\hline & \multicolumn{2}{|r|}{ Dice } \\
\hline & $\mathrm{ET}$ & $\mathrm{WT}$ TC \\
\hline Mean & 69.59 & 80.6875 .20 \\
\hline StdDev & 26.10 & 14.9328 .94 \\
\hline Median & 78.81 & 86.3887 .28 \\
\hline
\end{tabular}

Table 3. Results on BraTS 2020 test data. Metrics were computed by the online evaluation platform.

\section{Conclusion}

In this paper, we introduced a generic 3D U-Net architecture that allows performance transfer by re-using and embedding any $2 \mathrm{D}$ classifier network. The encoder as well as the decoder are composed of two stages. The 3D input data goes through a process of depth shrinking in order to transform the 3D data into $2 \mathrm{D}$ data. This process is a succession of blocks of $3 \mathrm{D}$ convolutions and maxpooling reducing the third dimension only. The transformed output data can be then encoded by any 2D classification network. Moreover, decoding also goes through a $2 \mathrm{D}$ decoding phase followed by a $3 \mathrm{D}$ decoding procedure. Because of the limited computational resources, we resized the images and trained separately the fourth modalities using four modality-specific networks. Nevertheless, the preliminary results seem to be promising. 
A. Belaid et al.
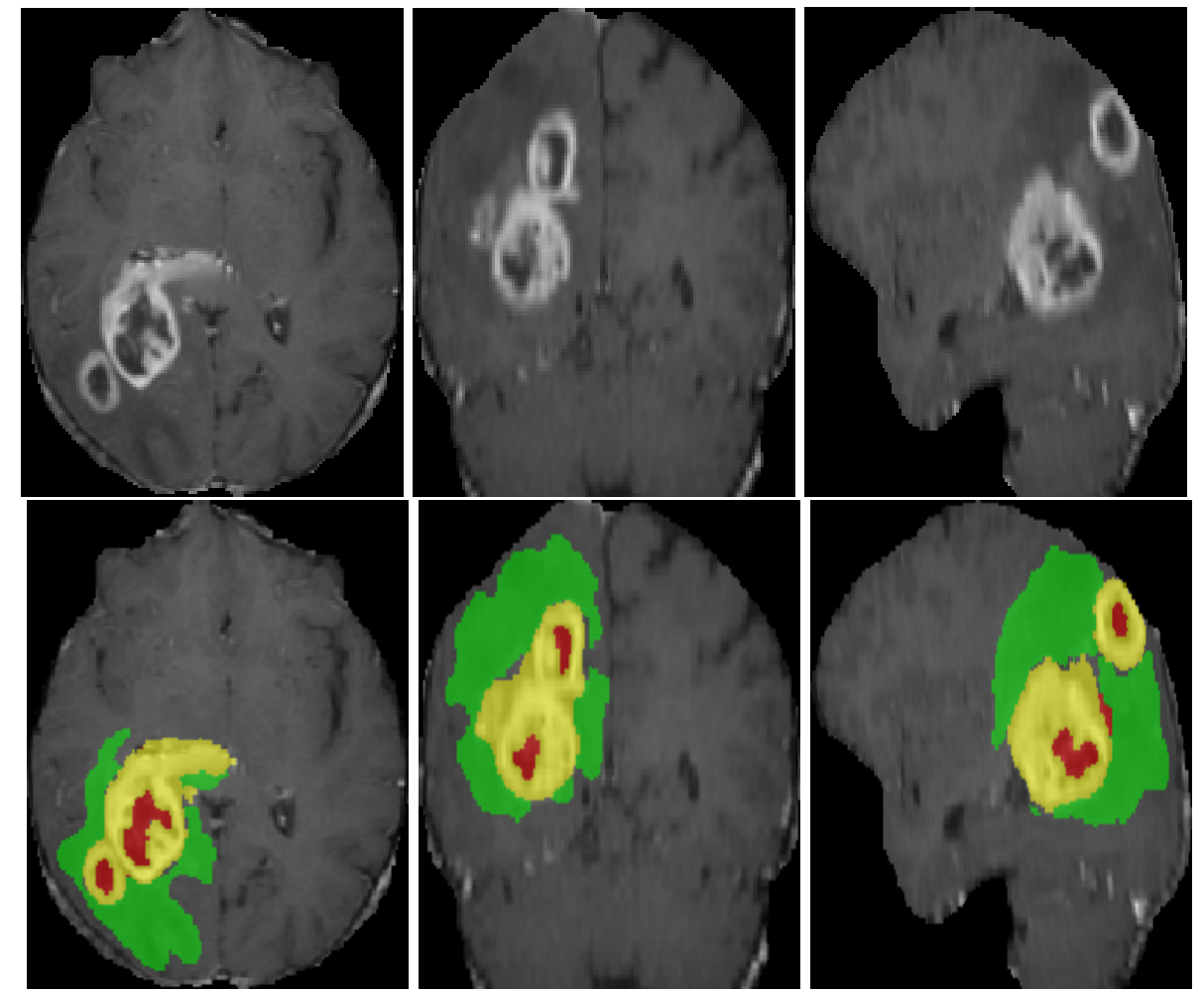

Fig. 2. Typical result on the BraTS validation set (2020). From left to right: axial, coronal end sagittal views in T1ce. Enhancing tumor is shown in yellow, necrosis in red and edema in green. 

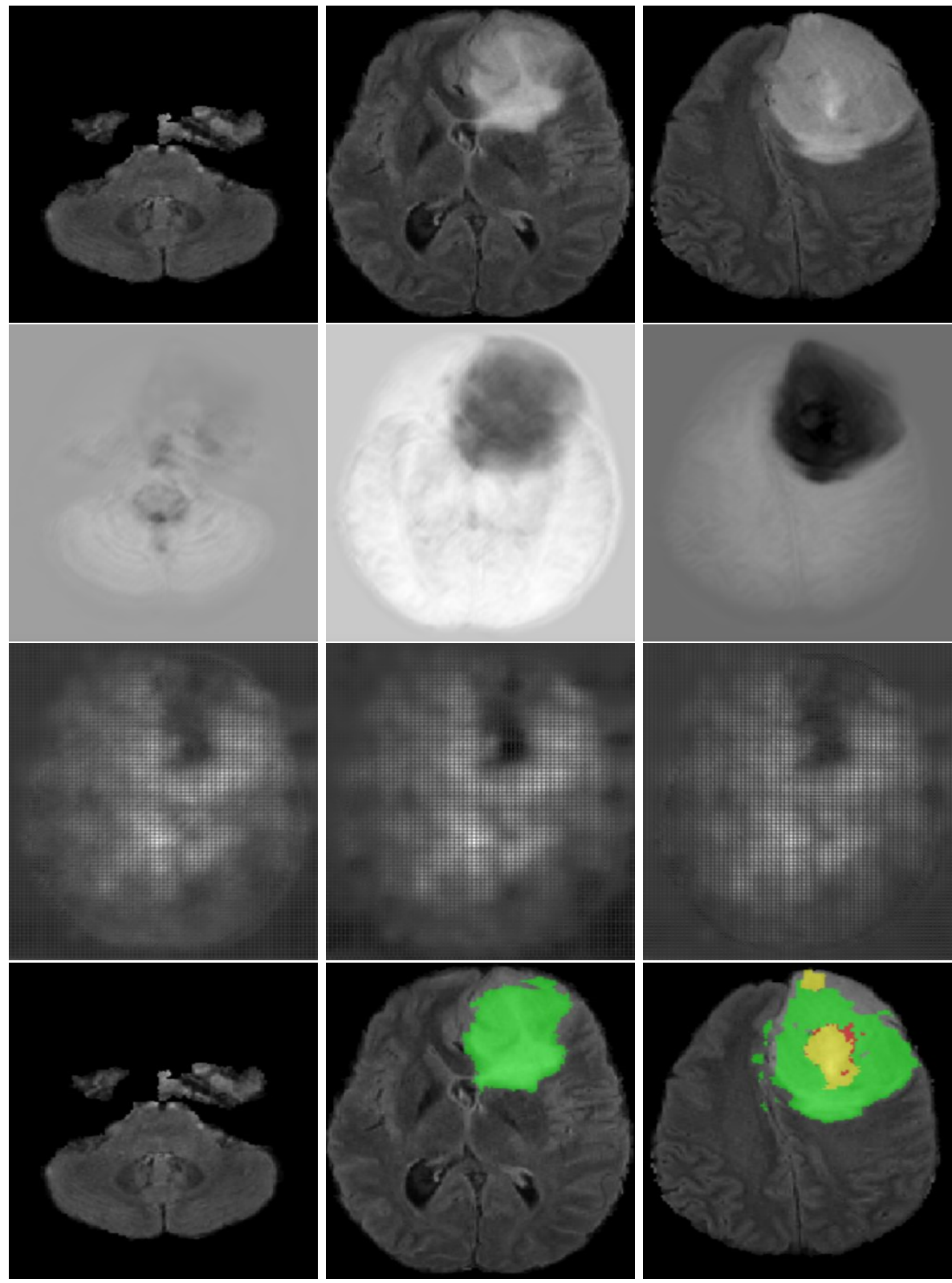

Fig. 3. Result visualization from the BraTS training set (2020). From up to down : three axial slices of an unique sample in Flair, corresponding channels output of the 3D encoder, corresponding channels input of the 3D decoder and corresponding labels. With Necrotic and Non-Enhancing Tumor core in red, Gadolinium-enhancing tumor in blue and peritumoral edema in green 

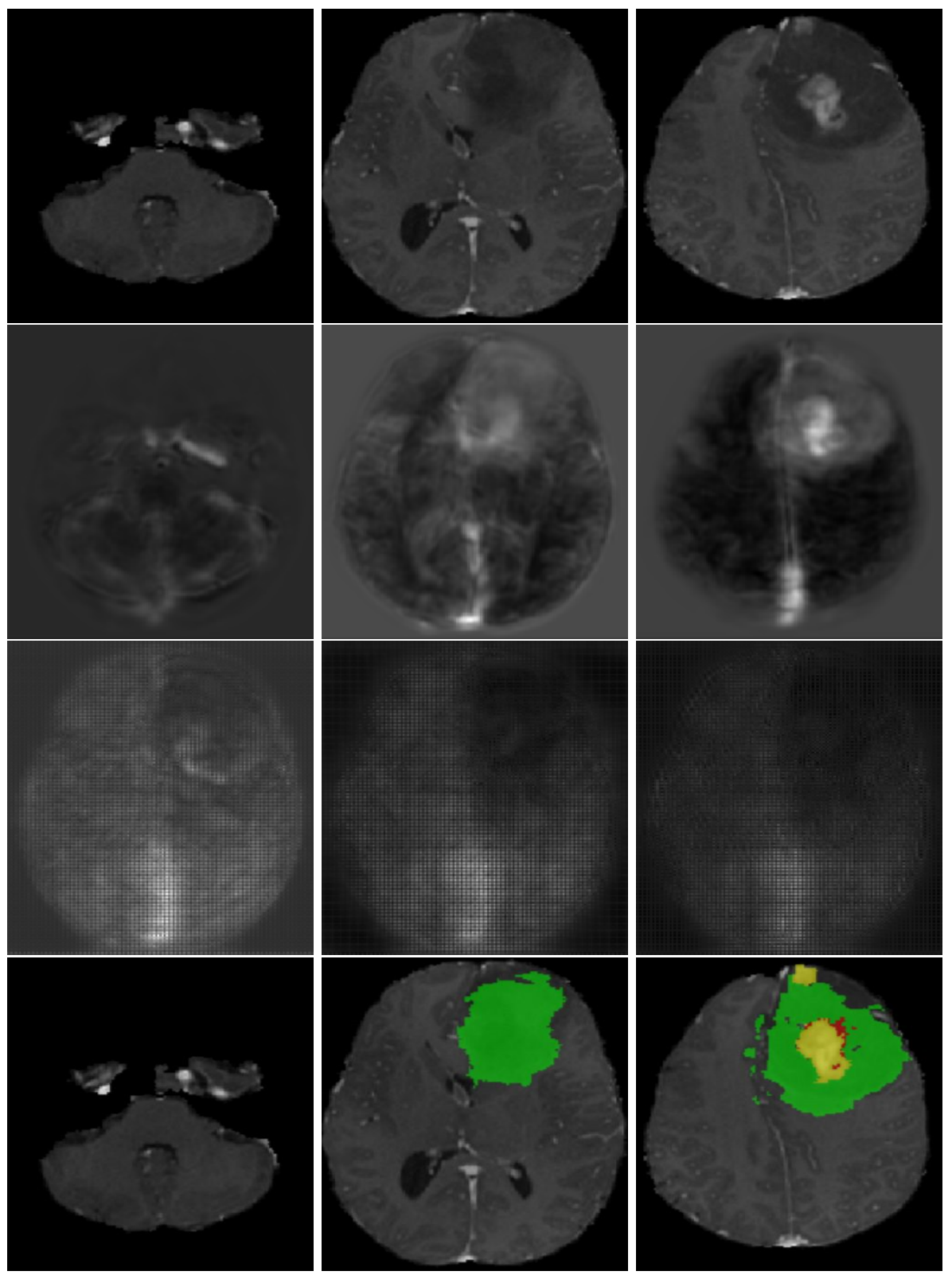

Fig. 4. Result visualization from the BraTS training set (2020). Three axial slices of an unique sample in T1ce, corresponding channels output of the 3D encoder, corresponding channels input of the 3D decoder and corresponding labels. With Necrotic and NonEnhancing Tumor core in red, Gadolinium-enhancing tumor in blue and peritumoral edema in green 
Our goal was not to surpass all the BraTS sophisticated segmentation techniques, but to provide a functional way to re-use $2 \mathrm{D}$ classification architectures for 3D medical image segmentation purposes. As can be seen, the learning transfer from weights trained on 2D natural images can be exploited for processing 3D medical images. We are convinced that we can significantly improve the results by robustifying the learning technique, keeping the original size of the data and stacking the 4 modalities together.

Acknowledgements This work has been sponsored by the General Directorate for Scientific Research and Technological Development, Ministry of Higher Education and Scientific Research (DGRSDT), Algeria.

\section{References}

1. S. Bakas, H. Akbari, A. Sotiras, M. Bilello, M. Rozycki, and J. Kirby et al. Segmentation labels and radiomic features for the pre-operative scans of the tcga-gbm collection. The Cancer Imaging Archive, 2017. DOI: 10.7937/K9/TCIA.2017.KLXWJJ1Q.

2. S. Bakas, H. Akbari, A. Sotiras, M. Bilello, M. Rozycki, and J. Kirby et al. Segmentation labels and radiomic features for the pre-operative scans of the tcga-gbm collection. The Cancer Imaging Archive, 2017. DOI: 10.7937/K9/TCIA.2017.GJQ7R0EF.

3. S. Bakas, H. Akbari, A. Sotiras, M. Bilello, M. Rozycki, and J.S. Kirby et al. Advancing the cancer genome atlas glioma mri collections with expert segmentation labels and radiomic features. Nature Scientific Data, page 4:170117, 2017. DOI: 10.1038/sdata.2017.117.

4. S. Bakas, M. Reyes, A. Jakab, S. Bauer, M. Rempfler, and A. Crimi et al. Identifying the best machine learning algorithms for brain tumor segmentation, progression assessment, and overall survival prediction in the brats challenge. arXiv preprint arXiv:1811.02629, 2018.

5. Stefan Bauer, Roland Wiest, Lutz-P Nolte, and Mauricio Reyes. A survey of mribased medical image analysis for brain tumor studies. Phys Med Biol., 58(13):R97R129, 2013. DOI: 10.1088/0031-9155/58/13/R97.

6. Pierre-Henri Conze, Sylvain Brochard, Valérie Burdin, Frances T.Sheehan, and Christelle Pons. Healthy versus pathological learning transferability in shoulder muscle MRI segmentation using deep convolutional encoderdecoders. Comput. Med. Imaging Graph., 83:101733, 2020. DOI: 10.1016/j.compmedimag.2020.101733.

7. Pierre-Henri Conze, Ali Emre Kavur, Emilie Cornec-Le Gall, Naciye Sinem Gezer, Yannick Le Meur, M. Alper Selver, and François Rousseau. Abdominal multi-organ segmentation with cascaded convolutional and adversarial deep networks. arXiv preprint arXiv:2001.09521, 2020.

8. B. H. Menze, A. Jakab, S. Bauer, J. Kalpathy-Cramer, K. Farahani, and J. Kirby et al. The multimodal brain tumor image segmentation benchmark (BRATS). IEEE Transactions on Medical Imaging, 34(10):1993-2024, 2015. DOI: 10.1109/TMI.2014.2377694.

9. Andriy Myronenko. 3D MRI brain tumor segmentation using autoencoder regularization.BrainLes@MICCAI, 2:311-320,2018. DOI:10.1007/978-3-030-11726-9 28 . 
10. O. Ronneberger, P. Fischer, and T. Brox. U-net: Convolutional networks for biomedical image segmentation. In International Conference on Medical Image Computing and Computer-Assisted Intervention, pages 234-241, 2015. 10.1007/978-3-319-24574-4 28.

11. K. Souadih, A. Belaid, D. Ben Salem, and P. H. Conze. Automatic forensic identification using 3D sphenoid sinus segmentation and deep characterization. Med. Biol. Eng. Comput., 58:291-306, 2020. DOI: 10.1007/s11517-019-02050-6.

12. Mingxing Tan and Quoc V. Le. Efficientnet: Rethinking model scaling for convolutional neural networks. In Proceedings of Machine Learning Research, editor, 36th International Conference on Machine Learning (ICML), volume 97, pages 10691-10700, Long Beach, California, USA, 2019.

13. Minh H. Vu, Guus Grimbergen, Tufve Nyholm, and Tommy Löfstedt. Evaluation of multi-slice inputs to convolutional neural networks for medical image segmentation. arXiv preprint arXiv:1912.09287, 2019.

14. Y. Wu and K. He. Group normalization. In In: European Conference on Computer Vision (ECCV), 2018. DOI: 10.1007/s11263-019-01198-w.

15. R. Zaouche, A. Belaid, S. Aloui, B. Solaiman, L. Lecornu, D. Ben Salem, and S. Tliba. Semi-automatic method for low-grade gliomas segmentation in magnetic resonance imaging. IRBM, 39(2):116-128, 2018. DOI: 10.1016/j.irbm.2018.01.004. 